\title{
MIXED JACOBI-LIKE FORMS OF SEVERAL VARIABLES
}

\author{
MIN HO LEE \\ Received 4 November 2005; Accepted 26 March 2006
}

We study mixed Jacobi-like forms of several variables associated to equivariant maps of the Poincaré upper half-plane in connection with usual Jacobi-like forms, Hilbert modular forms, and mixed automorphic forms. We also construct a lifting of a mixed automorphic form to such a mixed Jacobi-like form.

Copyright (c) 2006 Hindawi Publishing Corporation. All rights reserved.

\section{Introduction}

Jacobi-like forms of one variable are formal power series with holomorphic coefficients satisfying a certain transformation formula with respect to the action of a discrete subgroup $\Gamma$ of $\operatorname{SL}(2, \mathbb{R})$, and they are related to modular forms for $\Gamma$, which of course play a major role in number theory. Indeed, by using this transformation formula, it can be shown that that there is a one-to-one correspondence between Jacobi-like forms whose coefficients are holomorphic functions on the Poincare upper half-plane and certain sequences of modular forms of various weights (cf. [1, 12]). More precisely, each coefficient of such a Jacobi-like form can be expressed in terms of derivatives of a finite number of modular forms in the corresponding sequence. Jacobi-like forms are also closely linked to pseudodifferential operators, which are formal Laurent series for the formal inverse $\partial^{-1}$ of the differentiation operator $\partial$ with respect to the given variable (see, e.g., [1]). In addition to their natural connections with number theory and pseudodifferential operators, Jacobi-like forms have also been found to be related to conformal field theory in mathematical physics in recent years (see $[2,10]$ ).

The generalization of Jacobi-like forms to the case of several variables was studied in [8] in connection with Hilbert modular forms, which are essentially modular forms of several variables. As it is expected, Jacobi-like forms of several variables correspond to sequences of Hilbert modular forms. Another type of generalization can be provided by considering mixed Jacobi-like forms of one variable for a discrete subgroup $\Gamma \subset \operatorname{SL}(2, \mathbb{R})$, which are associated to a holomorphic map of the Poincaré upper half-plane that is equivariant with respect to a homomorphism of $\Gamma$ into $\operatorname{SL}(2, \mathbb{R})(\mathrm{cf} .[7,9])$. Mixed Jacobi-like

Hindawi Publishing Corporation

International Journal of Mathematics and Mathematical Sciences

Volume 2006, Article ID 31542, Pages 1-14

DOI 10.1155/IJMMS/2006/31542 
forms are related to mixed automorphic forms, and examples of mixed automorphic forms include holomorphic forms of the highest degree on the fiber product of elliptic surfaces (see [6]).

In this paper, we study mixed Jacobi-like forms of several variables associated to equivariant maps of the Poincaré upper half-plane in connection with usual Jacobi-like forms, Hilbert modular forms, and mixed automorphic forms. We also construct a lifting of a mixed automorphic form to such a mixed Jacobi-like form.

\section{Jacobi-like forms}

In this section, we review Jacobi-like forms of several variables and describe some of their properties. We also describe Hilbert modular forms, which are closely linked to such Jacobi-like forms.

Throughout this paper, we fix a positive integer $n$. Let $\left(z_{1}, \ldots, z_{n}\right)$ be the standard coordinate system for $\mathbb{C}^{n}$, and denote the associated partial differentiation operators by

$$
\partial_{1}=\frac{\partial}{\partial z_{1}}, \ldots, \partial_{n}=\frac{\partial}{\partial z_{n}} .
$$

We will often use the multi-index notation. Thus, given $\alpha=\left(\alpha_{1}, \ldots, \alpha_{n}\right) \in \mathbb{Z}^{n}$ and $u=$ $\left(u_{1}, \ldots, u_{n}\right) \in \mathbb{C}^{n}$, we have

$$
\partial^{\alpha}=\partial_{1}^{\alpha_{1}} \ldots \partial_{n}^{\alpha_{n}}, \quad u^{\alpha}=u_{1}^{\alpha_{1}} \ldots u_{n}^{\alpha_{n}}
$$

and for $\beta=\left(\beta_{1}, \ldots, \beta_{n}\right) \in \mathbb{Z}^{n}$, we write $\alpha \leq \beta$ if $\alpha_{i} \leq \beta_{i}$ for each $i=1, \ldots, n$. Furthermore, we also write $c=(c, \ldots, c) \in \mathbb{Z}^{n}$ if $c \in \mathbb{Z}$, and denote by $\mathbb{Z}_{+}$the set of nonnegative integers. Given $\alpha \in \mathbb{Z}^{n}$ and $\beta \in \mathbb{Z}_{+}^{n}$, we write $\beta !=\beta_{1}$ ! .. $\beta_{n}$ ! and

$$
\left(\begin{array}{l}
\alpha \\
\beta
\end{array}\right)=\left(\begin{array}{l}
\alpha_{1} \\
\beta_{1}
\end{array}\right) \cdots\left(\begin{array}{c}
\alpha_{n} \\
\beta_{n}
\end{array}\right)
$$

where for $1 \leq i \leq n$, we have $\left(\begin{array}{c}\alpha_{i} \\ 0\end{array}\right)=1$ and

$$
\left(\begin{array}{c}
\alpha_{i} \\
\beta_{i}
\end{array}\right)=\frac{\alpha_{i}\left(\alpha_{i}-1\right) \cdots\left(\alpha_{i}-\beta_{i}+1\right)}{\beta_{i} !}
$$

for $\beta_{i}>0$.

Let $\mathscr{H} \subset \mathbb{C}$ be the Poincare upper half-plane. Then the usual action of $\operatorname{SL}(2, \mathbb{R})$ on $\mathscr{H}$ by linear fractional transformations induces an action of $\operatorname{SL}(2, \mathbb{R})^{n}$ on the product $\mathscr{H}^{n}$ of $n$ copies of $\mathcal{H}$. Thus, if $\gamma \in \operatorname{SL}(2, \mathbb{R})^{n}$ and $z=\left(z_{1}, \ldots, z_{n}\right) \in \mathscr{H}^{n}$ with

$$
\gamma=\left(\gamma_{1}, \ldots, \gamma_{n}\right), \quad \gamma_{i}=\left(\begin{array}{ll}
a_{i} & b_{i} \\
c_{i} & d_{i}
\end{array}\right) \in \operatorname{SL}(2, \mathbb{R}) \quad(1 \leq i \leq n),
$$

then we have

$$
\gamma z=\left(\gamma_{1} z_{1}, \ldots, \gamma_{n} z_{n}\right)=\left(\frac{a_{1} z_{1}+b_{1}}{c_{1} z_{1}+d_{1}}, \ldots, \frac{a_{n} z_{n}+b_{n}}{c_{n} z_{n}+d_{n}}\right) \in \mathscr{H}^{n}
$$


For such $\gamma$ and $z$, we set

$$
J(\gamma, z)=\left(j\left(\gamma_{1}, z_{1}\right), \ldots, j\left(\gamma_{n}, z_{n}\right)\right) \in \mathbb{C}^{n}, \quad j\left(\gamma_{i}, z_{i}\right)=c_{i} z_{i}+d_{i}
$$

for $1 \leq i \leq n$. We denote by $\tilde{J}(\gamma, z)$ the diagonal matrix with diagonal entries $j\left(\gamma_{i}, z_{i}\right)$ with $1 \leq i \leq n$, that is,

$$
\tilde{J}(\gamma, z)=\operatorname{diag}\left(j\left(\gamma_{1}, z_{1}\right), \ldots, j\left(\gamma_{n}, z_{n}\right)\right) .
$$

Then the map $(\gamma, z) \mapsto \tilde{J}(\gamma, z)$ satisfies the cocycle condition

$$
\tilde{J}\left(\gamma \gamma^{\prime}, z\right)=\tilde{J}\left(\gamma, \gamma^{\prime} z\right) \tilde{J}\left(\gamma^{\prime}, z\right)
$$

for all $\gamma, \gamma^{\prime} \in \operatorname{SL}(2, \mathbb{R})^{n}$ and $z \in \mathscr{H}^{n}$. Given an element $\eta=\left(\eta_{1}, \ldots, \eta_{n}\right) \in \mathbb{Z}^{n}$ and a map $f: \mathscr{H}^{n} \rightarrow \mathbb{C}$, we set

$$
\left(\left.f\right|_{\eta} \gamma\right)(z)=J(\gamma, z)^{-\eta} f(\gamma z)
$$

for all $z \in \mathscr{H}^{n}$ and $\gamma \in \operatorname{SL}(2, \mathbb{R})^{n}$. Let $\Gamma$ be a discrete subgroup of $\operatorname{SL}(2, \mathbb{R})^{n}$.

Definition 2.1. Given $\eta=\left(\eta_{1}, \ldots, \eta_{n}\right) \in \mathbb{Z}_{+}^{n}$, a Hilbert modular form of weight $\eta$ for $\Gamma$ is a holomorphic function $f: \mathscr{H}^{n} \rightarrow \mathbb{C}$ such that

$$
\left.f\right|_{\eta} \gamma=f
$$

for all $\gamma \in \Gamma$, where $\left.f\right|_{\eta} \gamma$ is as in (2.10). Denote by $M_{\eta}(\Gamma)$ the space of all Hilbert modular forms of weight $\eta$ for $\Gamma$.

Remark 2.2. The usual definition of Hilbert modular forms also includes the regularity condition at the cusps, which is satisfied automatically for $n>1$ according to Koecher's principle (cf. $[3,4])$.

We denote by $R$ the ring of holomorphic functions $f\left(z_{1}, \ldots, z_{n}\right)$ on $\mathscr{H}^{n}$ and by $R[[X]]=$ $R\left[\left[X_{1}, \ldots, X_{n}\right]\right]$ the set of all formal power series in $X_{1}, \ldots, X_{n}$ with coefficients in $R$. Thus, using the multi-index notation, an element of $R[[X]]$ can be written in the form

$$
\Phi(z, X)=\sum_{\alpha \geq 0} f_{\alpha}(z) X^{\alpha}
$$

with $z=\left(z_{1}, \ldots, z_{n}\right) \in \mathscr{H}^{n}$ and $X^{\alpha}=X_{1}^{\alpha_{1}} \ldots X_{n}^{\alpha_{n}}$ for $\alpha=\left(\alpha_{1}, \ldots, \alpha_{n}\right) \in \mathbb{Z}_{+}^{n}$.

Let $\mathbb{C}^{\times}=\mathbb{C}-\{0\}$ be the set of nonzero complex numbers. Given $\lambda=\left(\lambda_{1}, \ldots, \lambda_{n}\right) \in$ $\left(\mathbb{C}^{\times}\right)^{n}$, we denote by $\tilde{\lambda}=\operatorname{diag}\left(\lambda_{1}, \ldots, \lambda_{n}\right)$ the associated $n \times n$ diagonal matrix, and set

$$
\mathbb{C}^{\times} X=\left\{X \tilde{\lambda} \mid \lambda \in\left(\mathbb{C}^{\times}\right)^{n}\right\}=\left\{\left(\lambda_{1} X_{1}, \ldots, \lambda_{n} X_{n}\right) \mid \lambda_{1}, \ldots, \lambda_{n} \in \mathbb{C}^{\times}\right\},
$$

where $X=\left(X_{1}, \ldots, X_{n}\right)$ is regarded as a row vector. Using (2.9), we see that $\operatorname{SL}(2, \mathbb{R})^{n}$ acts on $\mathscr{H}^{n} \times \mathbb{C}^{\times} X$ by

$$
\gamma \cdot(z, X \tilde{\lambda})=\left(\gamma z, X \tilde{J}(\gamma, z)^{-2} \tilde{\lambda}\right)
$$


4 Mixed Jacobi-like forms

for all $z \in \mathscr{H}^{n}, \lambda \in\left(\mathbb{C}^{\times}\right)^{n}$, and $\gamma \in \operatorname{SL}(2, \mathbb{R})^{n}$, where $\tilde{J}(\gamma, z)$ is as in $(2.8)$ so that

$$
X \tilde{J}(\gamma, z)^{-2} \tilde{\lambda}=\left(j\left(\gamma_{1}, z_{1}\right)^{-2} \lambda_{1} X_{1}, \ldots, j\left(\gamma_{n}, z_{n}\right)^{-2} \lambda_{n} X_{n}\right) .
$$

We now set

$$
K_{\xi, \eta}(\gamma,(z, X \tilde{\lambda}))=J(\gamma, z)^{\xi} \exp \left(\sum_{i=1}^{n} c_{i} \eta_{i} j\left(\gamma_{i}, z_{i}\right)^{-1} \lambda_{i} X_{i}\right)
$$

for $z \in \mathscr{H}^{n}, \gamma$ as in $(2.5)$, and $\lambda \in\left(\mathbb{C}^{\times}\right)^{n}$. Then it can be shown that

$$
K_{\xi, \eta}\left(\gamma \gamma^{\prime},(z, X \tilde{\lambda})\right)=K_{\xi, \eta}\left(\gamma, \gamma^{\prime} \cdot(z, X \tilde{\lambda})\right) K_{\xi, \eta}\left(\gamma^{\prime},(z, X \tilde{\lambda})\right)
$$

for all $\gamma, \gamma^{\prime} \in \operatorname{SL}(2, \mathbb{R})^{n}$, where $\gamma^{\prime} \cdot(z, X \tilde{\lambda})$ is as in $(2.14)$.

Definition 2.3. Given $\xi, \eta \in \mathbb{Z}^{n}$, a Jacobi-like form for $\Gamma$ of $n$ variables of weight $\xi$, and index $\eta$ is an element,

$$
\Phi(z, X)=\Phi\left(z, X_{1}, \ldots, X_{n}\right)
$$

of $R[[X]]$ satisfying

$$
\Phi\left(\gamma z, X \tilde{J}(\gamma, z)^{-2}\right)=K_{\xi, \eta}(\gamma,(z, X)) \Phi(z, X)
$$

for all $\gamma \in \Gamma$ and $z \in \mathscr{H}^{n}$. Denote by $\mathscr{F}_{\xi, \eta}(\Gamma)$ the space of all Jacobi-like forms of $n$ variables for $\Gamma$ of weight $\xi$ and index $\eta$.

Remark 2.4. Jacobi-like forms of several variables in $\mathscr{S}_{\xi, \eta}(\Gamma)$ with $\xi=\mathbf{0}$ and $\eta=\mathbf{1}$ were considered in [8], while Jacobi-like forms of one variable with index 0 were studied in [12].

Proposition 2.5. Given $\varepsilon \in \mathbb{Z}_{+}^{n}$, consider a formal power series

$$
\Phi(z, X)=\sum_{\alpha \geq \varepsilon} \phi_{\alpha}(z) X^{\alpha} \in R[[X]] .
$$

Then the following conditions are equivalent.

(i) The power series $\Phi(z, X)$ is a Jacobi-like form belonging to $\mathscr{F}_{\xi, \eta}(\Gamma)$.

(ii) The coefficient functions $\phi_{\alpha}: \mathscr{H} \rightarrow \mathbb{C}$ satisfy

$$
\left(\left.\phi_{\alpha}\right|_{2 \alpha+\xi} \gamma\right)(z)=\sum_{\delta=0}^{\alpha-\varepsilon} \frac{1}{\delta !} \frac{c^{\delta} \eta^{\delta}}{J(\gamma, z)^{\delta}} \phi_{\alpha-\delta}(z)
$$

for all $z \in \mathscr{H}^{n}$ and $\alpha \geq \varepsilon$, where $\gamma \in \Gamma$ is as in (2.5) with $c=\left(c_{1}, \ldots, c_{n}\right)$.

(iii) There exist modular forms $f_{v} \in M_{2 v+\xi}(\Gamma)$ for $v \geq \varepsilon$ such that

$$
\phi_{\alpha}(z)=\sum_{\beta=0}^{\alpha-\varepsilon} \frac{\eta^{\beta}}{\beta !(2 \alpha+\xi-\beta-\varepsilon) !} \partial^{\beta} f_{\alpha-\beta}(z)
$$

for all $\alpha \geq \varepsilon$. 
Proof. The proposition can be proved by slightly modifying the proofs of [8, Lemma 4.2 and Theorem 4.4].

If $\Phi(z, X)=\sum_{\alpha \geq \varepsilon} \phi_{\alpha}(z) X^{\alpha} \in \mathscr{J}_{\xi, \eta}(\Gamma)$, then (2.21) implies that

$$
\left.\phi_{\varepsilon}\right|_{2 \varepsilon+\xi} \gamma=\phi_{\varepsilon}
$$

for all $\gamma \in \Gamma$; hence the initial coefficient $\phi_{\varepsilon}(z)$ of the formal power series $\Phi(z, X)$ is a Hilbert modular form of weight $2 \varepsilon+\xi$ for $\Gamma$. We set

$$
\mathscr{G}_{\xi, \eta}(\Gamma)_{\varepsilon}=X^{\varepsilon} \mathscr{F}_{\xi, \eta}(\Gamma),
$$

which is a subspace of $\mathscr{F}_{\xi, \eta}(\Gamma)$ consisting of the elements of the form $\sum_{\alpha \geq \varepsilon} \phi_{\alpha}(z) X^{\alpha}$.

Then we see that there is a linear map

$$
\mathfrak{F}: \mathscr{J}_{\xi, \eta}(\Gamma)_{\mathcal{\varepsilon}} \longrightarrow M_{2 \varepsilon+\xi}(\Gamma)
$$

sending an element of $\mathscr{F}_{\xi, \eta}(\Gamma)_{\varepsilon}$ to its coefficient of $X^{\varepsilon}$.

\section{Mixed Jacobi-like forms}

In this section, we discuss Jacobi-like forms of several variables associated to holomorphic maps of the Poincare upper half-plane $\mathscr{H}$ that are equivariant with respect to a discrete subgroup of SL $(2, \mathbb{R})$. Such Jacobi-like forms are related to mixed automorphic forms.

Let $\Gamma$ be a discrete subgroup of $\operatorname{SL}(2, \mathbb{R})$, and for each $k \in\{1, \ldots, n\}$, let $\omega_{k}: \mathscr{H} \rightarrow \mathscr{H}$ and $\chi_{k}: \Gamma \rightarrow \operatorname{SL}(2, \mathbb{R})$ be a holomorphic map and a group homomorphism, respectively, satisfying

$$
\omega_{k}(\gamma \zeta)=\chi_{k}(\gamma) \omega_{k}(\zeta)
$$

for all $\zeta \in \mathscr{H}$ and $\gamma \in \Gamma$. By setting

$$
\omega=\left(\omega_{1}, \ldots, \omega_{n}\right), \quad \chi=\left(\chi_{1}, \ldots, \chi_{n}\right),
$$

we obtain a holomorphic map $\omega: \mathscr{H} \rightarrow \mathbb{C}^{n}$ and a homomorphism $\chi: \Gamma \rightarrow \operatorname{SL}(2, \mathbb{R})^{n}$. Given $\eta=\left(\eta_{1}, \ldots, \eta_{n}\right) \in \mathbb{Z}^{n}$, we define the map $J_{\omega, \chi}: \operatorname{SL}(2, \mathbb{R}) \times \mathscr{H} \rightarrow \mathbb{C}^{n}$ by

$$
J_{\omega, \chi}(\gamma, \zeta)=\left(j\left(\chi_{1}(\gamma), \omega_{1}(\zeta)\right), \ldots, j\left(\chi_{n}(\gamma), \omega_{n}(\zeta)\right)\right)
$$

for all $\gamma \in \operatorname{SL}(2, \mathbb{R})$ and $\zeta \in \mathscr{H}$, where $j: \operatorname{SL}(2, \mathbb{R}) \times \mathscr{H} \rightarrow \mathbb{C}$ is as in (2.7).

Definition 3.1. Given $\xi=\left(\xi_{1}, \ldots, \xi_{n}\right) \in \mathbb{Z}^{n}$, a mixed automorphic form of type $\xi$ associated to $\Gamma, \omega$, and $\chi$ is a holomorphic map $f: \mathscr{H} \rightarrow \mathbb{C}$ satisfying

$$
f(\gamma \zeta)=J_{\omega, \chi}(\gamma, \zeta)^{\xi} f(\zeta)=j\left(\chi_{1}(\gamma), \omega_{1}(\zeta)\right)^{\xi_{1}} \ldots j\left(\chi_{n}(\gamma), \omega_{n}(\zeta)\right)^{\xi_{n}} f(\zeta)
$$

for all $\zeta \in \mathscr{H}$ and $\gamma \in \Gamma$. Denote by $M_{\xi}(\Gamma, \omega, \chi)$ the space of mixed automorphic forms of type $\xi$ associated to $\Gamma, \omega$, and $\chi$. 
Definition 3.2. Let $\mathscr{F}$ be the set of holomorphic functions on $\mathscr{H}$, and let $\mathscr{F}[[X]]$ be the space of formal power series in $X=\left(X_{1}, \ldots, X_{n}\right)$. Given $\xi=\left(\xi_{1}, \ldots, \xi_{n}\right), \eta=\left(\eta_{1}, \ldots, \eta_{n}\right) \in$ $\mathbb{Z}^{n}$, a formal power series $F(\zeta, X) \in \mathscr{F}[[X]]$ is a mixed Jacobi-like form of weight $\xi$ and index $\eta$ associated to $\Gamma$, $\omega$, and $\chi$ if it satisfies

$$
F\left(\gamma \zeta, X \tilde{J}_{\omega, \chi}(\gamma, \zeta)^{-2}\right)=J_{\omega, \chi}(\gamma, \zeta)^{\xi} \exp \left(\sum_{k=1}^{n} \frac{c_{\chi, k} \eta_{k} X_{k}}{j\left(\chi_{k}(\gamma), \omega_{k}(\zeta)\right)}\right) F(\zeta, X)
$$

for all $\zeta \in \mathscr{H}$ and $\gamma \in \Gamma$, where $\tilde{J}_{\omega, \chi}(\gamma, \zeta)$ denotes the diagonal matrix

$$
\operatorname{diag}\left(j\left(\chi_{1}(\gamma), \omega_{1}(\zeta)\right), \ldots, j\left(\chi_{n}(\gamma), \omega_{n}(\zeta)\right)\right)
$$

and $c_{\chi, k}$ is the $(2,1)$-entry of the matrix $\chi_{k}(\gamma) \in \operatorname{SL}(2, \mathbb{R})$. Denote by $\mathscr{F}_{\xi, \eta}(\Gamma, \omega, \chi)$ the space of mixed Jacobi-like forms of weight $\xi$ and index $\eta$ associated to $\Gamma, \omega$, and $\chi$.

Given $\mu \in \mathbb{Z}^{n}$ and a function $h: \mathscr{H} \rightarrow \mathbb{C}$, set

$$
\left(\left.h\right|_{\mu} ^{\omega, \chi} \gamma\right)(\zeta)=h(\gamma \zeta) J_{\omega, \chi}(\gamma, \zeta)^{-\mu}
$$

for all $\zeta \in \mathscr{H}$ and $\gamma \in \Gamma$.

Lemma 3.3. A formal power series $F(\zeta, X)=\sum_{\alpha \geq \varepsilon} f_{\alpha}(\zeta) X^{\alpha} \in \mathscr{F}[[X]]$ with $\varepsilon \in \mathbb{Z}_{+}^{n}$ is an element of $\mathscr{S}_{\xi, \eta}(\Gamma, \omega, \chi)$ if and only if

$$
\left(\left.f_{\alpha}\right|_{2 \alpha+\xi} ^{\omega, \chi} \gamma\right)(\zeta)=\sum_{\delta=0}^{\alpha-\varepsilon} \frac{1}{\delta !} \frac{c_{\chi}^{\delta} \eta^{\delta}}{J_{\omega, \chi}(\gamma, \zeta)^{\delta}} f_{\alpha-\delta}(\zeta)
$$

for all $\gamma \in \Gamma$ with $c_{\chi}=\left(c_{\chi, 1}, \ldots, c_{\chi, n}\right), \zeta \in \mathscr{H}^{n}$, and $\alpha \geq \varepsilon$, where $c_{\chi, j}$ denotes the $(2,1)$-entry of the matrix $\chi_{j}(\gamma) \in \operatorname{SL}(2, \mathbb{R})$ for $1 \leq j \leq n$. In particular, the initial coefficient $f_{\varepsilon}(\zeta)$ of $F(\zeta, X)$ is an element of $M_{2 \varepsilon+\xi}(\Gamma, \omega, \chi)$ if $F(\zeta, X) \in \mathscr{F}_{\xi, \eta}(\Gamma, \omega, \chi)$.

Proof. Given $\gamma \in \Gamma$ as described by (3.4) and (3.5), the formal power series $F(\zeta, X)=$ $\sum_{\alpha \geq \varepsilon} f_{\alpha}(\zeta) X^{\alpha}$ is an element of $\mathscr{F}_{\xi, \eta}(\Gamma, \omega, \chi)$ if and only if

$$
\begin{aligned}
\sum_{\alpha \geq \varepsilon} f_{\alpha}(\gamma \zeta) J_{\omega, \chi}(\gamma, \zeta)^{-2 \alpha-\xi} X^{\alpha} & =\prod_{i=1}^{n}\left(\sum_{\mu_{i}=0}^{\infty} \frac{1}{\mu_{i} !} \frac{c_{\chi, i}^{\mu_{i}} \eta_{i}^{\mu_{i}} X_{i}^{\mu_{i}}}{j\left(\chi_{i}(\gamma), \omega_{i}(z)\right)^{\mu_{i}}}\right) \cdot \sum_{\nu \geq \varepsilon} f_{\nu}(\zeta) X^{\nu} \\
& =\sum_{\mu \geq 0} \sum_{\nu \geq \varepsilon} \frac{1}{\mu !} \frac{c^{\mu} \eta^{\mu}}{J_{\omega, \chi}(\gamma, \zeta)^{\mu}} f_{\nu}(\zeta) X^{\mu+\nu}
\end{aligned}
$$

for all $\zeta \in \mathscr{H}$. Thus by comparing the coefficients of $X^{\alpha}$, we obtain

$$
f_{\alpha}(\gamma \zeta) J_{\omega, \chi}(\gamma, \zeta)^{-2 \alpha-\xi}=\sum_{\delta=0}^{\alpha-\varepsilon} \frac{1}{\delta !} \frac{c^{\delta} \eta^{\delta}}{J_{\omega, \chi}(\gamma, \zeta)^{\delta}} f_{\alpha-\delta}(\zeta)
$$

and therefore the lemma follows. 
For each $\varepsilon \in \mathbb{Z}^{n}$ with $\varepsilon \geq \mathbf{0}$, we set

$$
\mathscr{g}_{\xi, \eta}(\Gamma, \omega, \chi)_{\varepsilon}=X^{\varepsilon} \mathscr{g}_{\xi, \eta}(\Gamma, \omega, \chi)
$$

Then by Lemma 3.3, we see that there is a linear map

$$
\mathscr{F}_{\omega, \chi}: \mathscr{F}_{\xi, \eta}(\Gamma, \omega, \chi)_{\varepsilon} \longrightarrow M_{2 \varepsilon+\xi}(\Gamma, \omega, \chi)
$$

sending an element $\sum_{\alpha \geq \varepsilon} f_{\alpha}(\zeta) X^{\alpha}$ of $\mathscr{F}_{\xi, \eta}(\Gamma, \omega, \chi)$ to its initial coefficient $f_{\varepsilon}(\zeta)$.

If $R$ is the set of holomorphic functions on $\mathscr{H}^{n}$ as in Section 2, we define the maps

$$
\Delta^{\omega}: R \longrightarrow \mathscr{F}, \quad \Delta_{X}^{\omega}: R[[X]] \longrightarrow \mathscr{F}[[X]]
$$

associated to the map $\omega: \mathscr{H} \rightarrow \mathscr{H}^{n}$ as in (3.2) by

$$
\left(\Delta^{\omega} h\right)(\zeta)=h(\omega(\zeta)), \quad\left(\Delta_{X}^{\omega} F\right)(\zeta, X)=F(\omega(\zeta), X)
$$

for all $\zeta \in \mathscr{H}, h \in R$, and $F \in R[[X]]$. Given a discrete subgroup $\Gamma$ of $\operatorname{SL}(2, \mathbb{R})$, let $\widetilde{\Gamma}_{\chi}$ be a discrete subgroup of $\operatorname{SL}(2, \mathbb{R})^{n}$ such that

$$
\chi(\Gamma)=\chi_{1}(\Gamma) \times \cdots \times \chi_{n}(\Gamma) \subset \widetilde{\Gamma}_{\chi}
$$

where $\chi=\left(\chi_{1}, \ldots, \chi_{n}\right)$ is as in (3.2).

Theorem 3.4. (i) If $\Delta^{\omega}: R \rightarrow \mathscr{F}$ and $\Delta_{X}^{\omega}: R[[X]] \rightarrow \mathscr{F}[[X]]$ are as in (3.14), then

$$
\Delta^{\omega}\left(M_{\xi}\left(\tilde{\Gamma}_{\chi}\right)\right) \subset M_{\xi}(\Gamma, \omega, \chi), \quad \Delta_{X}^{\omega}\left(\mathscr{F}_{\xi, \eta}\left(\tilde{\Gamma}_{\chi}\right)_{\varepsilon}\right) \subset \mathscr{F}_{\xi, \eta}(\Gamma, \omega, \chi)_{\varepsilon}
$$

for all $\xi, \eta \in \mathbb{Z}^{n}$.

(ii) If $\mathscr{F}_{F}$ and $\mathscr{F}_{\omega, \chi}$ are the linear maps in (2.25) and (3.12), respectively, then the diagram



is commutative.

Proof. If $f: \mathscr{H}^{n} \rightarrow \mathbb{C}$ is an element of $\mathcal{M}_{\xi}\left(\widetilde{\Gamma}_{\chi}\right)$, then by (3.14) we have

$$
\begin{aligned}
\left(\Delta^{\omega} f\right)(\gamma \zeta) & =f(\omega(\gamma \zeta))=f\left(\chi_{1}(\gamma) \omega_{1}(\zeta), \ldots, \chi_{n}(\gamma) \omega_{n}(\zeta)\right) \\
& =J(\chi(\gamma), \omega(\zeta))^{\xi} f(\omega(\zeta))=J(\chi(\gamma), \omega(\zeta))^{\xi}\left(\Delta^{\omega} f\right)(\zeta)
\end{aligned}
$$


for all $\zeta \in \mathscr{H}$ and $\gamma \in \Gamma$; hence $\Delta^{\omega} f$ is an element of $M_{\xi}\left(\widetilde{\Gamma}_{\chi}, \omega, \chi\right)$. On the other hand, if $F$ is an element of $\mathscr{F}_{\xi, \eta}\left(\widetilde{\Gamma}_{\chi}\right)$ by (3.5) and (3.14), we see that

$$
\begin{aligned}
\left(\Delta_{X}^{\omega}(F)\right)\left(\gamma \zeta, X \widetilde{J}_{\omega, \chi}(\gamma, \zeta)^{-2}\right) & =F\left(\chi_{1}(\gamma) \omega_{1}(\zeta), \ldots, \chi_{n}(\gamma) \omega_{n}(\zeta), X \widetilde{J}_{\omega, \chi}(\gamma, \zeta)^{-2}\right) \\
& =J(\chi(\gamma), \omega(\zeta))^{\xi} \exp \left(\sum_{k=1}^{n} \frac{c_{\chi, k} \eta_{k} X_{k}}{j\left(\chi_{k}(\gamma), \omega_{k}(\zeta)\right)}\right) F(\omega(\zeta), X)
\end{aligned}
$$

for all $\zeta \in \mathscr{H}$ and $\gamma \in \Gamma$. Thus $\Delta_{X}^{\omega} F$ is an element of $\mathscr{F}_{\xi, \eta}(\Gamma, \omega, \chi)$, and $\Delta_{X}^{\omega} F \in \mathscr{F}_{\xi, \eta}(\Gamma, \omega, \chi)_{\varepsilon}$ if $F \in \mathscr{S}_{\xi, \eta}\left(\widetilde{\Gamma}_{\chi}\right)_{\mathcal{E}}$, which proves (i). In order to verify (ii), consider an element $\Phi(\zeta, X)=$ $\sum_{\alpha \geq \varepsilon} \phi_{\alpha}(\zeta) X^{\alpha} \in \mathscr{g}_{\xi, \eta}\left(\widetilde{\Gamma}_{\chi}\right)_{\varepsilon}$. Then we have

$$
\left(\left(\Delta^{\omega} \circ \mathscr{F}\right)(\Phi)\right)(\zeta)=\left(\Delta^{\omega} \phi_{\varepsilon}\right)(\zeta)=\phi_{\varepsilon}\left(\omega_{1}(\zeta), \ldots, \omega_{n}(\zeta)\right)
$$

for $\zeta \in \mathscr{H}$. On the other hand, we have

$$
\begin{aligned}
\left(\Delta_{X}^{\omega} \Phi\right)(\zeta, X) & =\Phi(\omega(\zeta), X)=\Phi\left(\omega_{1}(\zeta), \ldots, \omega_{n}(\zeta), X\right) \\
& =\sum_{\alpha \geq \varepsilon} \phi_{\alpha}\left(\omega_{1}(\zeta), \ldots, \omega_{n}(\zeta)\right) X^{\alpha}
\end{aligned}
$$

Thus we see that

$$
\left(\left(\mathscr{F}_{\omega, \chi} \circ \Delta_{X}^{\omega}\right)(\Phi)\right)(\zeta)=\phi_{\varepsilon}\left(\omega_{1}(\zeta), \ldots, \omega_{n}(\zeta)\right)=\left(\left(\Delta^{\omega} \circ \mathscr{F}\right)(\Phi)\right)(\zeta)
$$

which implies (ii); hence the proof of the theorem is complete.

\section{Examples}

In this section, we discuss two examples related to mixed Jacobi-like forms. The first one involves a fiber bundle over a Riemann surface whose generic fiber is the product of elliptic curves, and the second one is linked to solutions of linear ordinary differential equations.

Example 4.1. Let $E$ be an elliptic surface (cf. [5]). Thus $E$ is a compact surface over $\mathbb{C}$ that is the total space of an elliptic fibration $\pi: E \rightarrow X$ over a Riemann surface $X$. Let $E_{0}$ be the union of the regular fibers of $\pi$, and let $\Gamma \subset \operatorname{PSL}(2, \mathbb{R})$ be the fundamental group of $X_{0}=\pi\left(E_{0}\right)$. Then the universal covering space of $X_{0}$ may be identified with the Poincare upper half-plane $\mathscr{H}$, and we have $X_{0}=\Gamma \backslash \mathcal{H}$, where $\Gamma$ is regarded as a subgroup of $\operatorname{SL}(2, \mathbb{R})$ and the quotient is taken with respect to the action given by linear fractional transformations. Given $z \in \mathcal{H}_{0}$, let $\Phi$ be a holomorphic 1-form on $E_{z}=\pi^{-1}(z)$, and choose an ordered basis $\left\{\alpha_{1}(z), \alpha_{2}(z)\right\}$ for $H_{1}\left(E_{z}, \mathbb{Z}\right)$ which depends on the parameter $z$ in a continuous manner. If we set

$$
\omega_{1}(z)=\int_{\alpha_{1}(z)} \Phi, \quad \omega_{2}(z)=\int_{\alpha_{2}(z)} \Phi
$$


then $\omega_{1} / \omega_{2}$ is a many-valued function from $X_{0}$ to $\mathscr{H}$ which can be lifted to a single-valued function $\omega: \mathscr{H} \rightarrow \mathscr{H}$ on the universal cover $\mathscr{H}$ of $X_{0}$. Then it can be shown that there is a group homomorphism $\chi: \Gamma \rightarrow \operatorname{SL}(2, \mathbb{R})$, called the monodromy representation for the elliptic surface $E$, such that

$$
\omega(\gamma z)=\chi(\gamma) \omega(z)
$$

for all $\gamma \in \Gamma$ and $z \in \mathscr{H}$. Thus the maps $\chi$ and $\omega$ form an equivariant pair.

Let $\left(\chi_{j}, \omega_{j}\right)$ be an equivariant pair associated to an elliptic surface $E$ of the type described above for each $j \in\{1, \ldots, p\}$, and set

$$
\tilde{\chi}=\left(1, \chi_{1}, \ldots, \chi_{p}\right), \quad \widetilde{\omega}=\left(1, \omega_{1}, \ldots, \omega_{p}\right) .
$$

Then, given a positive integer $p$ and an element $\mathbf{m}=\left(m_{1}, \ldots, m_{p}\right) \in \mathbb{Z}^{q}$ with $m_{1}, \ldots, m_{p}>$ 0 , the semidirect product $\Gamma \ltimes \tilde{\chi}\left(\mathbb{Z}^{2}\right)^{|\mathbf{m}| p}$ with $|\mathbf{m}|=m_{1}+\cdots+m_{p}$ associated to $\tilde{\chi}$ acts on $\mathscr{H} \times \mathbb{C}^{|\mathbf{m}| p}$ by

$$
\left(\gamma, \boldsymbol{\ell}_{1}, \ldots, \boldsymbol{\ell}_{p}\right) \cdot\left(z, \zeta_{1}, \ldots, \boldsymbol{\zeta}_{p}\right)=\left(\gamma z, \hat{\zeta}_{1}, \ldots, \hat{\zeta}_{p}\right)
$$

for all $\gamma \in \Gamma$ and $z \in \mathscr{H}$, where

$$
\begin{aligned}
& \boldsymbol{\ell}_{j}=\left(\left(\mu_{1, j}, \nu_{1, j}\right), \ldots,\left(\mu_{m_{j}, j}, \nu_{m_{j}, j}\right)\right) \in\left(\mathbb{Z}^{2}\right)^{m_{j}}, \\
& \boldsymbol{\zeta}_{j}=\left(\zeta_{1, j}, \ldots, \zeta_{m_{j}, j}\right), \hat{\zeta}_{j}=\left(\hat{\zeta}_{1, j}, \ldots, \hat{\zeta}_{m_{j}, j}\right) \in \mathbb{C}^{m_{j}}
\end{aligned}
$$

for $1 \leq j \leq p$ with

$$
\hat{\zeta}_{r, j}=\frac{\zeta_{r, j}+\omega_{j}(z) \mu_{r, j}+v_{r, j}}{c_{\chi_{j}} \omega_{j}(z)+d_{\chi_{j}}}
$$

for each $r \in\left\{1, \ldots, m_{j}\right\}$ if

$$
\chi_{j}(\gamma)=\left(\begin{array}{ll}
a_{\chi_{j}} & b_{\chi_{j}} \\
c_{\chi_{j}} & d_{\chi_{j}}
\end{array}\right) \in \operatorname{SL}(2, \mathbb{R}) .
$$

We denote by $E_{0}^{|\mathbf{m}|}$ the associated quotient space, that is,

$$
E_{0}^{|\mathbf{m}| p}=\Gamma \times\left(\mathbb{Z}^{2}\right)^{|\mathbf{m}| p} \backslash \mathscr{H} \times \mathbb{C}^{|\mathbf{m}| p} .
$$

Given $\varepsilon \in \mathbb{Z}^{p+1}$, we set $\xi=\left(2, m_{1}, \ldots, m_{p}\right)-2 \varepsilon$, and let $F(z, X) \in \mathscr{G}_{\xi, \eta}(\Gamma, \boldsymbol{\omega}, \boldsymbol{\chi})_{\varepsilon}$. Then by Lemma 3.3, we see that $\mathscr{F}_{\omega, \chi}(F(z, X))$ is an element of $\mathcal{M}_{\left(2, m_{1}, \ldots, m_{p}\right)}(\Gamma, \omega, \chi)$, and it can be shown that the associated holomorphic form

$$
\omega_{F}(\mathbf{z})=\mathscr{F}_{\omega, \chi}(F(z, X)) d z \wedge d \zeta_{1} \wedge \cdots \wedge d \zeta_{p}
$$

on $\mathscr{H} \times \mathbb{C}^{|\mathbf{m}| p}$ with $\mathbf{z}=\left(z, \zeta_{1}, \ldots, \zeta_{p}\right) \in \mathscr{H} \times \mathbb{C}^{|\mathbf{m}| p}$ is invariant under the action of $\Gamma \times$ $\left(\mathbb{Z}^{2}\right)^{p}$. Hence $\omega_{F}(\mathbf{z})$ can be regarded as a holomorphic $(|\mathbf{m}| p+1)$-form on $E_{0}^{|\mathbf{m}| p}$, and 
therefore we obtain a canonical map

$$
\mathscr{J}_{\xi, \eta}(\Gamma, \boldsymbol{\omega}, \boldsymbol{\chi})_{\varepsilon} \longrightarrow \Omega^{p+1}\left(E_{0}^{|\mathbf{m}| p}\right)
$$

from $\mathscr{S}_{\xi, \eta}(\Gamma, \boldsymbol{\omega}, \boldsymbol{\chi})_{\varepsilon}$ to the space $\Omega^{p+1}\left(E_{0}^{|\mathbf{m}| p}\right)$ of holomorphic $(|\mathbf{m}| p+1)$-forms on $E_{0}^{|\mathbf{m}| p}$.

Example 4.2. Let $\Gamma$ be a Fuchsian group of the first kind, and let $K(X)$ be the function field of the smooth complex algebraic curve $X=\Gamma \backslash \mathcal{H} \cup\{$ cusps $\}$. Consider a second-order linear differential equation

$$
\left(\frac{d^{2}}{d x^{2}}+\widetilde{P}(x) \frac{d}{d x}+\widetilde{Q}(x)\right) \tilde{f}=0
$$

for $x \in X$ and $\widetilde{P}(x), \widetilde{Q}(x) \in K(X)$ with regular singular points, whose singular points are contained in $\Gamma \backslash\{$ cusps $\} \subset X$. Let

$$
\Lambda f=\left(\frac{d^{2}}{d z^{2}}+P(z) \frac{d}{d z}+Q(z)\right) f=0
$$

for $z \in \mathscr{H}$, be the differential equation obtained by pulling back (4.11) via the natural projection $\mathscr{H} \rightarrow \Gamma \backslash \mathcal{H} \subset X$. Let $\sigma_{1}$ and $\sigma_{2}$ be linearly independent solutions of (4.12), and let $S^{m}(\Lambda)$ be the linear ordinary differential operator of order $m+1$ such that the $m+1$ functions

$$
\sigma_{1}^{m}, \sigma_{1}^{m-1} \sigma_{2}, \ldots, \sigma_{1} \sigma_{2}^{m-1}, \sigma_{2}^{m}
$$

are linearly independent solutions of the corresponding linear homogeneous equation $S^{m}(\Lambda) f=0$. Let $\chi: \Gamma \rightarrow \operatorname{SL}(2, \mathbb{R})$ be the monodromy representation of $\Gamma$ for the secondorder equation $\Lambda f=0$. Then the period map $\omega: \mathscr{H} \rightarrow \mathscr{H}$ defined by $\omega(z)=\sigma_{1}(z) / \sigma_{2}(z)$ for all $z \in \mathscr{H}$ is equivariant with respect to $\chi$. Let $\psi: \mathscr{H} \rightarrow \mathbb{C}$ be a function corresponding to an element of $K(X)$ satisfying the parabolic residue condition in the sense of [11, Definition 3.20], and let $f^{\psi}$ be a solution of the nonhomogeneous equation $S^{m}(\Lambda) f=\psi$. Then the function

$$
\frac{d^{m+1}}{d \omega(z)^{m+1}}\left(\frac{f^{\psi}(z)}{\sigma_{2}(z)^{m}}\right)
$$

is a mixed automorphic form of type $(0, m+2)$ associated to $\Gamma, \omega$, and $\chi$ (cf. [11, page 32]).

Given a positive integer $p$ and $\mathbf{m}=\left(m_{1}, \ldots, m_{p}\right) \in \mathbb{Z}^{p}$ with $m_{1}, \ldots, m_{p}>0$, we consider a system of ordinary differential equations

$$
S^{m_{j}}\left(\Lambda_{j}\right) f_{j}\left(z_{j}\right)=\psi_{j}\left(z_{j}\right), \quad 1 \leq j \leq p,
$$

of the type described above and for each $j \in\{1, \ldots, p\}$, choose a solution $f_{j}^{\psi_{j}}\left(z_{j}\right)$ for the $j$ th equation. For $1 \leq j \leq p$, let $\chi_{j}: \Gamma_{j} \rightarrow \operatorname{SL}(2, \mathbb{R})$ and $\omega_{j}: \mathscr{H} \rightarrow \mathcal{H}$ be the monodromy representation and the period map, respectively, associated to the operator $S^{m_{j}}\left(\Lambda_{j}\right)$, and 
set

$$
\tilde{\chi}=\left(\chi_{1}, \ldots, \chi_{p}\right), \quad \tilde{\omega}=\left(\omega_{1}, \ldots, \omega_{p}\right), \quad \Gamma=\Gamma_{1} \cap \cdots \cap \Gamma_{p} .
$$

Then we see that the function $\hat{f}: \mathscr{H} \rightarrow \mathbb{C}$ defined by

$$
\widehat{f}(z)=f_{1}(z) \cdots f_{p}(z)
$$

for all $z \in \mathscr{H}$ is a mixed automorphic form belonging to $M_{\mathbf{m}}(\Gamma, \tilde{\omega}, \tilde{\chi})$.

\section{Liftings of mixed automorphic forms}

Let $\omega=\left(\omega_{1}, \ldots, \omega_{n}\right)$ and $\chi=\left(\chi_{1}, \ldots, \chi_{n}\right)$ be as in Section 3. Thus $\omega_{i}: \mathscr{H} \rightarrow \mathscr{H}$ is a holomorphic map equivariant with respect to the homomorphism $\chi_{i}: \Gamma \rightarrow \operatorname{SL}(2, \mathbb{R})$ for each $i \in\{1, \ldots, n\}$, where $\Gamma$ is a discrete subgroup of $\operatorname{SL}(2, \mathbb{R})$. In this section, we construct liftings of mixed automorphic forms associated to $\Gamma, \omega$, and $\chi$ of certain types to mixed Jacobi-like forms associated to $\Gamma, \omega$, and $\chi$.

We first consider discrete subgroups $\Gamma_{1}, \ldots, \Gamma_{n}$ of $\operatorname{SL}(2, \mathbb{R})$ satisfying

$$
\chi_{i}(\Gamma) \subset \Gamma_{i}
$$

for all $i \in\{1, \ldots, n\}$. Given $\xi=\left(\xi_{1}, \ldots, \xi_{n}\right) \in \mathbb{Z}^{n}$ and $\mu=\left(\mu_{1}, \ldots, \mu_{n}\right) \in \mathbb{Z}_{+}^{n}$, let $M_{2 \mu_{i}+\xi_{i}}\left(\Gamma_{i}\right)$ denote the space of automorphic forms of one variable for $\Gamma_{i}$ of weight $2 \mu_{i}+\xi_{i}$. If $\Delta^{\omega_{i}}$ is the map in (3.14) associated to $\omega_{i}: \mathscr{H} \rightarrow \mathscr{H}$ in the case of $n=1$, then we see that

$$
\Delta^{\omega_{i}}\left(M_{2 \mu_{i}+\xi_{i}}\left(\Gamma_{i}\right)\right)=\left\{h \circ \omega_{i} \mid h \in M_{2 \mu_{i}+\xi_{i}}\left(\Gamma_{i}\right)\right\}
$$

for $1 \leq i \leq n$. We denote the tensor product of these spaces by

$$
M_{2 \mu+\xi}^{0}(\Gamma, \omega, \chi)=\bigotimes_{i=1}^{n} \Delta^{\omega_{i}}\left(M_{2 \mu_{i}+\xi_{i}}\left(\Gamma_{i}\right)\right),
$$

and consider an element of the form

$$
\mathfrak{h}=\sum_{k=1}^{p} C_{k} \bigotimes_{i=1}^{n}\left(h_{i, k} \circ \omega_{i}\right) \in \mathcal{M}_{2 \mu+\xi}^{0}(\Gamma, \omega, \chi)
$$

with $C_{k} \in \mathbb{C}$ and $h_{i, k} \in M_{2 \mu_{i}+\xi_{i}}\left(\Gamma_{i}\right)$ for $1 \leq i \leq n$ and $1 \leq k \leq p$. Then we have

$$
\begin{aligned}
\mathfrak{h}(\gamma z) & =\sum_{k=1}^{p} C_{k} \bigotimes_{i=1}^{n}\left(h_{i, k}\left(\omega_{i}(\gamma z)\right)\right)=\sum_{k=1}^{p} C_{k} \bigotimes_{i=1}^{n}\left(h_{i, k}\left(\chi_{i}(\gamma) \omega_{i}(z)\right)\right) \\
& =\sum_{k=1}^{p} C_{k} \bigotimes_{i=1}^{n}\left(j\left(\chi_{i}(\gamma), \omega_{i}(z)\right)^{2 \mu_{i}+\xi_{i}} h_{i, k}\left(\chi_{i}(\gamma) \omega_{i}(z)\right)\right) \\
& =\left(\prod_{i=1}^{n} j\left(\chi_{i}(\gamma), \omega_{i}(z)\right)^{2 \mu_{i}+\xi_{i}}\right) \mathfrak{h}(z)
\end{aligned}
$$


for all $z \in \mathscr{H}$ and $\gamma \in \Gamma$; hence $\mathfrak{h}$ is a mixed automorphic form belonging to $M_{2 \mu+\xi}(\Gamma, \omega, \chi)$. Thus we see that $M_{2 \mu+\xi}^{0}(\Gamma, \omega, \chi)$ is a subspace of $M_{2 \mu+\xi}(\Gamma, \omega, \chi)$.

We now discuss a lifting of an element of $\mathcal{M}_{2 \mu+\xi}^{0}(\Gamma, \omega, \chi)$ to a Jacobi-like form belonging to $\mathscr{S}_{\xi, \eta}(\Gamma, \omega, \chi)_{\varepsilon}$ with $\varepsilon=\left(\varepsilon_{1}, \ldots, \varepsilon_{n}\right) \in \mathbb{Z}_{+}^{n}$ and $\eta=\left(\eta_{1}, \ldots, \eta_{n}\right) \in \mathbb{Z}^{n}$. Given $i \in\{1, \ldots, n\}$ and $k \in\{1, \ldots, p\}$, assuming that $\mu \geq \varepsilon$, we set

$$
\hat{h}_{i, k, \ell}=\frac{\eta_{i}^{\ell-\mu_{i}} h_{i, k}^{\left(\ell-\mu_{i}\right)}}{\left(\ell-\mu_{i}\right) !\left(\ell+\xi_{i}+\mu_{i}-\varepsilon_{i}\right) !}
$$

for $\ell \geq \mu_{i}$ and

$$
\hat{h}_{k, \alpha}^{\omega}(z)=\left(\hat{h}_{1, k, \alpha_{1}}\left(\omega_{1}(z)\right), \ldots, \hat{h}_{n, k, \alpha_{n}}\left(\omega_{n}(z)\right)\right)
$$

for all $z \in \mathscr{H}$ and $\alpha=\left(\alpha_{1}, \ldots, \alpha_{n}\right) \geq \mu$. We define the formal power series $\Phi_{\mathfrak{h}}(z, X) \in$ $R[[X]]$ associated to $\mathfrak{h}$ by

$$
\Phi_{\mathfrak{h}}(z, X)=\sum_{k=1}^{p} C_{k} \sum_{\alpha \geq \mu}\left(\hat{h}_{k, \alpha}^{\omega}(z)\right)^{\mathbf{1}} X^{\alpha}
$$

where $\mathbf{1}=(1, \ldots, 1) \in \mathbb{Z}^{n}$ so that

$$
\left(\hat{h}_{k, \alpha}^{\omega}(z)\right)^{1}=\hat{h}_{1, k, \alpha_{1}}\left(\omega_{1}(z)\right) \cdots \hat{h}_{n, k, \alpha_{n}}\left(\omega_{n}(z)\right)
$$

for $\alpha=\left(\alpha_{1}, \ldots, \alpha_{n}\right)$.

Theorem 5.1. The map $\mathfrak{h} \mapsto \Phi_{\mathfrak{h}}$ determines a lifting of an element of $\mathcal{M}_{2 \mu+\xi}^{0}(\Gamma, \omega, \chi)$ to a Jacobi-like form belonging to $\mathscr{S}_{\xi, \eta}(\Gamma, \omega, \chi)_{\mu} \subset \mathscr{J}_{\xi, \eta}(\Gamma, \omega, \chi)_{\varepsilon}$ such that

$$
\mathscr{F}_{\omega, \chi}\left(\Phi_{\mathfrak{h}}\right)=\frac{\mathfrak{h}}{(2 \mu+\xi-\varepsilon) !}
$$

for all $\mathfrak{h} \in \mathcal{M}_{2 \mu+\xi}^{0}(\Gamma, \omega, \chi)$, where $\mathscr{F}_{\omega, \chi}$ is the map sending $\Phi_{\mathfrak{h}}(z, X)$ to the coefficient of $X^{\mu}$ as in (3.12).

Proof. For $1 \leq i \leq n$, applying Proposition 2.5 to the case of $n=1$, we see that the formal power series

$$
\Phi_{i}\left(z, X_{i}\right)=\sum_{\ell \geq \varepsilon_{i}} \phi_{\ell}(z) X_{i}^{\ell}
$$

in $X_{i}$ is a Jacobi-like form of one variable belonging to $\mathscr{E}_{\xi_{i}, \eta_{i}}\left(\Gamma_{i}\right)_{\mathcal{\varepsilon}_{i}}$ if and only if there is a sequence of modular forms $\left\{f_{r}\right\}_{r \geq 0}$ with $f_{r} \in M_{2 r+\xi_{i}}\left(\Gamma_{i}\right)$ satisfying

$$
\phi_{\ell}=\sum_{j=0}^{\ell-\varepsilon_{i}} \frac{\eta_{i}^{j} f_{\ell-j}^{(j)}}{j !\left(2 \ell+\xi_{i}-j-\varepsilon_{i}\right) !}
$$


for all $\ell \geq \varepsilon_{i}$. We now consider an element $\mathfrak{h} \in \mathcal{M}_{\varepsilon}^{0}(\Gamma, \omega, \chi)$ given by (5.4). Given $i$ and $k$, let $\left\{f_{r}\right\}_{r \geq 0}$ be the sequence of functions on $\mathscr{H}$ defined by

$$
f_{r}= \begin{cases}h_{i, k} & \text { if } r=\mu_{i} \\ 0 & \text { otherwise }\end{cases}
$$

Then clearly $f_{r} \in M_{2 r+\xi_{i}}\left(\Gamma_{i}\right)$ for each $r \geq \varepsilon_{i}$. If $\Phi_{i, k}\left(z, X_{i}\right)=\sum_{\ell \geq \varepsilon_{i}} \phi_{i, k, \ell}(z) X_{i}^{\ell}$ is the corresponding Jacobi-like form belonging to $\mathscr{E}_{\xi_{i}, \eta_{i}}\left(\Gamma_{i}\right)$, then by $(5.12)$ the coefficient function $\phi_{i, k, \ell}$ coincides with $\hat{h}_{i, k, \ell}$ in (5.6). Thus for each $k$, we see that the product

$$
\begin{aligned}
\Phi_{k}^{\omega}(z, X) & =\Phi_{1, k}\left(\omega_{1}(z), X_{1}\right) \cdots \Phi_{n, k}\left(\omega_{n}(z), X_{n}\right) \\
& =\sum_{\alpha_{1} \geq \mu_{1}} \cdots \sum_{\alpha_{n} \geq \mu_{n}} \hat{h}_{1, k, \alpha_{1}}\left(\omega_{1}(z)\right) \cdots \hat{h}_{n, k, \alpha_{n}}\left(\omega_{n}(z)\right) X_{1}^{\alpha_{1}} \cdots X_{n}^{\alpha_{n}} \\
& =\sum_{\alpha \geq \mu}\left(\hat{h}_{k, \alpha}^{\omega}(z)\right)^{1} X^{\alpha}
\end{aligned}
$$

is a Jacobi-like form belonging to $\mathscr{F}_{\xi, \eta}(\Gamma, \omega, \chi)_{\mu} \subset \mathscr{F}_{\xi, \eta}(\Gamma, \omega, \chi)_{\mathcal{\varepsilon}}$. From this and the fact that the formal power series in (5.8) can be written in the form

$$
\Phi_{\mathfrak{h}}(z, X)=\sum_{k=1}^{p} C_{k} \Phi_{k}^{\omega}(z, X)
$$

we see that $\Phi_{\mathfrak{h}}(z, X)$ is a Jacobi-like form belonging to $\mathscr{F}_{\xi, \eta}(\Gamma, \omega, \chi)_{\mu}$. On the other hand, from (5.6) we have

$$
\hat{h}_{i, k, \mu_{i}}=\frac{h_{i, k}}{\left(2 \mu_{i}+\xi_{i}-\varepsilon_{i}\right) !}
$$

for $1 \leq i \leq n$ and $1 \leq k \leq p$, which implies that

$$
\begin{aligned}
\mathscr{F}_{\omega, \chi}\left(\Phi_{k}^{\omega}(z, X)\right) & =\frac{h_{1, k}\left(\omega_{1}(z)\right) \cdots h_{n, k}\left(\omega_{n}(z)\right)}{\left(2 \mu_{1}+\xi_{1}-\varepsilon_{1}\right) ! \cdots\left(2 \mu_{n}+\xi_{n}-\varepsilon_{n}\right) !} \\
& =\frac{h_{1, k}\left(\omega_{1}(z)\right) \cdots h_{n, k}\left(\omega_{n}(z)\right)}{(2 \mu+\xi-\varepsilon) !} .
\end{aligned}
$$

Combining this with (5.15), we obtain

$$
\mathscr{F}_{\omega, \chi}\left(\Phi_{\mathfrak{h}}(z, X)\right)=\sum_{k=1}^{p} C_{k}\left(\frac{h_{1, k}\left(\omega_{1}(z)\right) \cdots h_{n, k}\left(\omega_{n}(z)\right)}{(2 \mu+\xi-\varepsilon) !}\right)=\frac{\mathfrak{h}(z)}{(2 \mu+\xi-\varepsilon) !}
$$

where we identified the tensor product with the usual product in $\mathbb{C}$; hence the proof of the theorem is complete. 


\section{Mixed Jacobi-like forms}

\section{References}

[1] P. B. Cohen, Y. Manin, and D. Zagier, Automorphic pseudodifferential operators, Algebraic Aspects of Integrable Systems, Progress in Nonlinear Differential Equations and Their Applications, vol. 26, Birkhäuser Boston, Massachusetts, 1997, pp. 17-47.

[2] C. Dong and G. Mason, Transformation laws for theta functions, Proceedings on Moonshine and Related Topics (Montréal, QC, 1999), CRM Proceedings \& Lecture Notes, vol. 30, American Mathematical Society, Rhode Island, 2001, pp. 15-26.

[3] E. Freitag, Hilbert Modular Forms, Springer, Berlin, 1990.

[4] P. B. Garrett, Holomorphic Hilbert Modular Forms, The Wadsworth \& Brooks/Cole Mathematics Series, Wadsworth, California, 1990.

[5] K. Kodaira, On compact analytic surfaces. II, Annals of Mathematics. Second Series 77 (1963), 563-626.

[6] M. H. Lee, Mixed cusp forms and holomorphic forms on elliptic varieties, Pacific Journal of Mathematics 132 (1988), no. 2, 363-370.

[7] _ Mixed Jacobi-like forms, Complex Variables. Theory and Application 42 (2000), no. 4, 387-396.

[8] _ Hilbert modular pseudodifferential operators, Proceedings of the American Mathematical Society 129 (2001), no. 11, 3151-3160.

[9] __ Mixed Automorphic Forms, Torus Bundles, and Jacobi Forms, Lecture Notes in Mathematics, vol. 1845, Springer, Berlin, 2004.

[10] M. Miyamoto, A modular invariance on the theta functions defined on vertex operator algebras, Duke Mathematical Journal 101 (2000), no. 2, 221-236.

[11] P. Stiller, Special values of Dirichlet series, monodromy, and the periods of automorphic forms, Memoirs of the American Mathematical Society 49 (1984), no. 299, iv+116.

[12] D. Zagier, Modular forms and differential operators, Proceedings of the Indian Academy of Sciences. Mathematical Sciences 104 (1994), no. 1, 57-75.

Min Ho Lee: Department of Mathematics, University of Northern Iowa, Cedar Falls, IA 50614-0506, USA

E-mail address: lee@math.uni.edu 


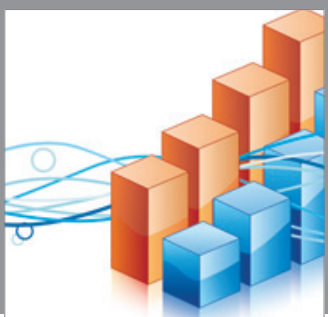

Advances in

Operations Research

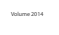

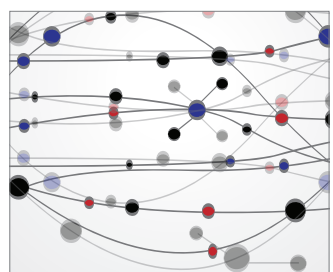

\section{The Scientific} World Journal
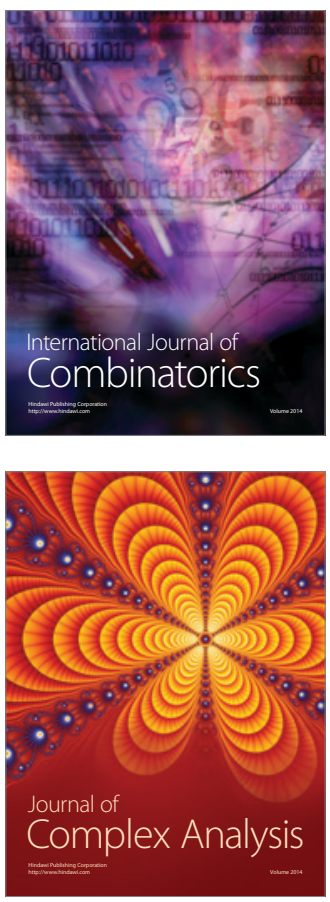

International Journal of

Mathematics and

Mathematical

Sciences
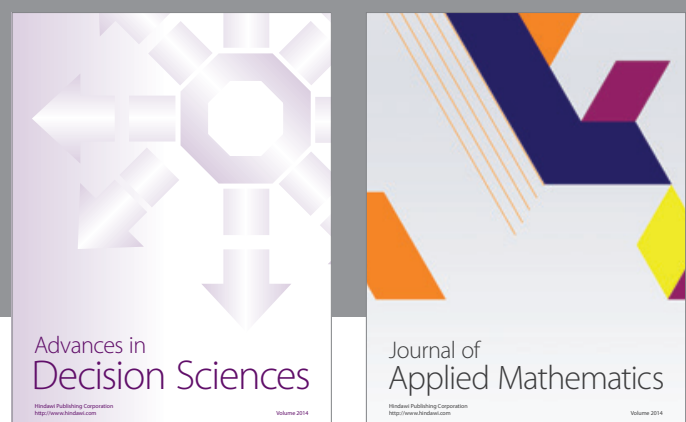

Journal of

Applied Mathematics


Submit your manuscripts at http://www.hindawi.com
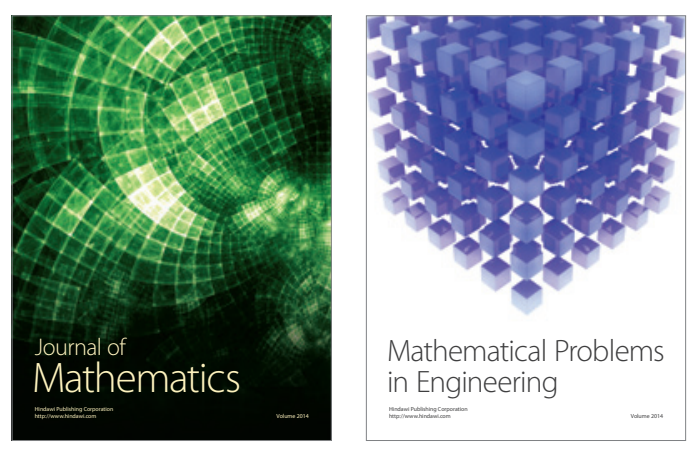

Mathematical Problems in Engineering
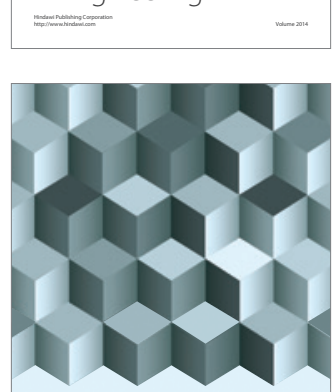

Journal of

Function Spaces
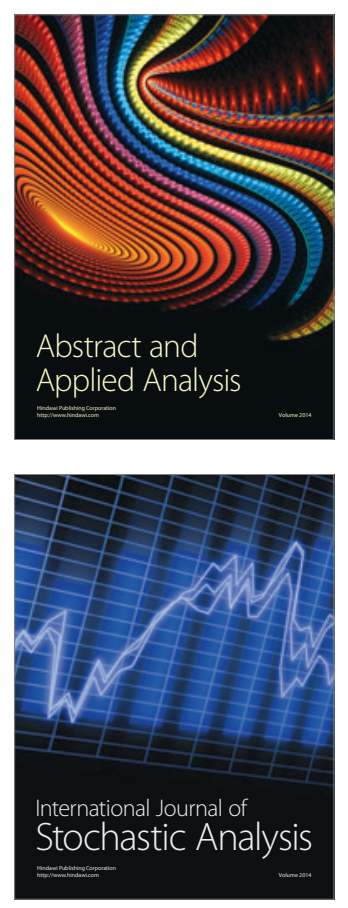



ournal of

Probability and Statistics

Promensencen
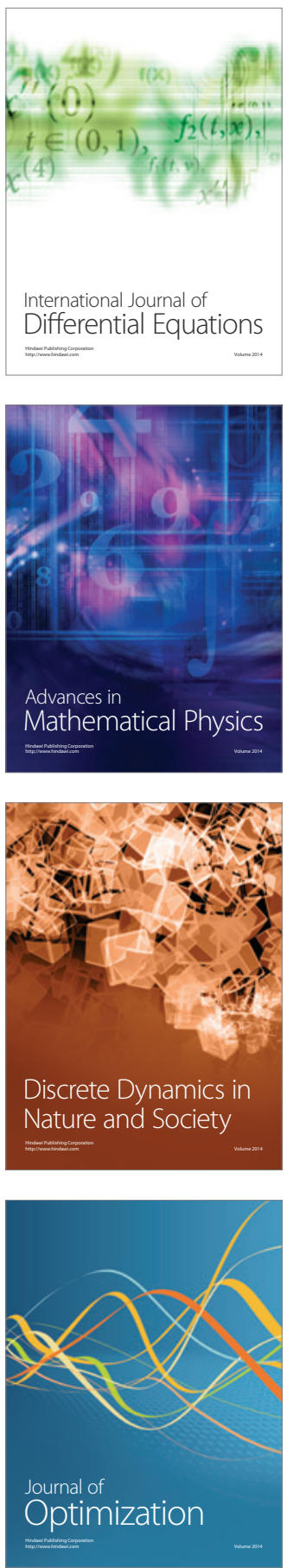